ISSN 1991-8631

Original Paper

http://indexmedicus.afro.who.int

\title{
Caractérisation physico-chimique et diversité phytoplanctonique des eaux du lac de Zowla (Lac Boko), Togo
}

\author{
Kossivi ATANLE ${ }^{1,2^{*}}$, Moctar L. BAWA ${ }^{1}$, Kouami KOKOU ${ }^{2}$ et \\ Gbandi DJANEYE-BOUNDJOU ${ }^{1}$
}

\author{
${ }^{1}$ Laboratoire de Chimie des Eaux, FDS, Université de Lomé, BP : 1515, Lomé, Togo. \\ ${ }^{2}$ Laboratoire de Botanique et Ecologie Végétale, FDS, Université de Lomé, BP : 1515, Lomé, Togo. \\ *Auteur correspondant, E-mail : lucatanle@yahoo.fr, Tel : (+228) 91902171
}

\section{RESUME}

Cette étude a été initiée pour évaluer l'impact de l'eau de mer et des cours d'eau sur les caractéristiques physico-chimiques et sur la diversité phytoplanctonique de l'eau du lac de Zowla. Pour ce faire, cinq sites de prélèvement dans le lac et cinq autres dans les rivières tributaires du lac ont été retenus en Juin 2007 correspondant à la grande saison pluvieuse et en Août 2007 correspondant à la petite saison sèche. Les paramètres physico-chimiques ont été déterminés par les méthodes AFNOR. L'identification des espèces de phytoplancton a été faite au microscope photonique ordinaire. L'étude a révélé une conductivité électrique très élevée d'une valeur maximale de $8370 \mu \mathrm{S} / \mathrm{cm}$ au site le plus proche de la mer en Juin, confirmée par exemple par une teneur en chlorure très élevée ( $2723 \mathrm{mg} / \mathrm{l}$ au même site en Juin). En Août, on note une oxydabilité au $\mathrm{KMnO}_{4}$ élevée (valeur maximale : $10 \mathrm{mgO}_{2} / \mathrm{l}$ ) aux sites le plus éloigné et le plus proche de la mer. S'agissant de la diversité de la flore algale, les espèces de la classe des diatomophycées sont les plus diversifiées au cours des deux saisons, mais cette suprématie est plus marquée en grande saison pluvieuse qu'en petite saison pluvieuse (80,55\% de la composition qualitative de la flore algale en Juin contre 40\% en Août). L'Analyse en Composantes principales (ACP) a montré que les cyanophycées sont plus diversifiées en espèces dans les milieux aquatiques turbides riches en éléments minéraux azotés et phosphatés, les euchlorophycées le sont dans les milieux très minéralisés et que les diatomophycées sont cosmopolites. Cette étude montre en outre que les paramètres physico-chimiques du lac de Zowla sont influencés en grande saison des pluies par l'eau de mer et en petite saison sèche par l'eau du fleuve Mono.

(C) 2012 International Formulae Group. All rights reserved.

Mots clés : Pollution, Paramètre physico-chimique, Phytoplancton, Lac.

\section{INTRODUCTION}

Le Togo par son réseau hydrographique dispose d'un nombre important d'écosystèmes d'eau douce dont la qualité ne cesse de se dégrader de jour en jour. Mais, peu d'études sont consacrées à la pollution de ces écosystèmes d'après des investigations menées. Pour ce qui concerne le phytoplancton et la pollution de l'eau, quelques études ont été réalisées dans une moindre mesure seulement dans la partie sud du pays (Edorh et al., 2008). Parmi ces cours d'eau, il y a le lac de Zowla qui semble très affecté par la pollution à cause, peut-être, de 
l'ouverture quasi-permanente de la passe entre la mer et le système lagunaire et du drainage par les rivières tributaires du lac des déchets de phosphate provenant de l'exploitation du minerai au sud du Togo. Or, selon Jiang et Shen (2006), une augmentation continue de la quantité de phosphore pourrait être le facteur principal d'eutrophisation d'un lac. Selon Gulati et Van Donk (2002), les apports externes d'éléments nutritifs (azote, phosphore) et les eaux polluées sont les causes principales d'eutrophisation des lacs. Ceci conduit à une augmentation de la biomasse phytoplanctonique constituée surtout d'algues microscopiques, à une eau trouble due aux phosphates et nitrates en suspension dans l'eau et à des changements biologiques indésirables. Wetzel et Likens (2000) définissent pour leur part le phénomène d'eutrophisation comme étant l'enrichissement d'un plan d'eau par des éléments nutritifs utiles à la croissance des plantes ou autres producteurs primaires souvent sous forme azotée ou phosphatée. Pour Chikhaoui et al. (2008), la disponibilité des nutriments constitue le principal facteur qui contrôle le phytoplancton lorsque les conditions de lumière et de température sont adéquates.

A l'instar des lacs pollués, celui de Zowla a perdu beaucoup d'atouts qu'il procurait aux populations riveraines dans leur vécu quotidien. C'est un lac qui les ravitaillait en produits halieutiques qu'elles commercialisaient. C'est un lac qui était favorable à la navigation, sur lequel on pouvait pratiquer le sport nautique, qui attirait beaucoup de touristes selon les enquêtes auprès des riverains. Aujourd'hui, beaucoup d'espèces animales et végétales y ont disparu car les changements biologiques occasionnés par la pollution des eaux d'un cours d'eau (Gulati et Van Donk, 2002) incluent le plus souvent la perte de biodiversité : la disparition de macrophytes submergés, des bouleversements dans la lignée des poissons et la diminution du contrôle, de la surface au fond, des zooplanctons sur les phytoplanctons
(Xie et Xie, 2002). L'appauvrissement du milieu dû à la dégradation de la qualité de l'eau du lac entraîne de jour en jour un exode rural massif et des problèmes de santé liés à la pollution de l'eau. La connaissance de l'état de pollution des eaux du lac peut alors amener à trouver des approches de solution.

Face à cette situation, cette étude a été initiée afin de connaître les impacts de la pollution sur les caractéristiques physicochimiques et la diversité phytoplanctonique de l'eau du lac. Elle permettra également d'établir une corrélation entre les paramètres physico-chimiques et la diversité du phytoplancton.

\section{MATERIEL ET METHODES Cadre d'étude}

Localisé dans la Région Maritime au Togo, le lac de Zowla est à cheval entre les Préfectures des Lacs et de Vo. Il mesure au maximum 5,5 km de long sur 2,1 km de large et a une superficie de $6,55 \mathrm{~km}^{2}$ selon le logiciel Mapinfo version 7.0. La profondeur maximale mesurée sur le site était comprise entre 1 et 1,5 m en Juin 2007. Il est rejoint au niveau de Zalivé par le lac de Togoville. Les deux lacs, par un couloir qui part de Zalivé, se jettent dans la mer à Aného. Le lac de Zowla est alimenté en eau douce par les rivières Boko, Elia, Hato et Voukpo (Figure 1), puis par le fleuve Mono avec lequel il est en communication à travers le chenal de la Gbaga qui rejoint le couloir à Aného. Il peut alors subir l'influence de l'eau de mer et de l'eau douce. Le bassin versant du lac de Zowla, à l'instar de toute la Région Maritime du Togo, jouit d'un climat de type subéquatorial ou guinéen à deux saisons de pluies alternant avec deux saisons sèches. Les principales activités économiques relevées dans les agglomérations sont la pêche, l'agriculture et/ou l'élevage.

Les données de ce travail proviennent de dix sites de prélèvement : cinq sites dans les rivières tributaires du lac et les autres dans le lac (Figure 1). 


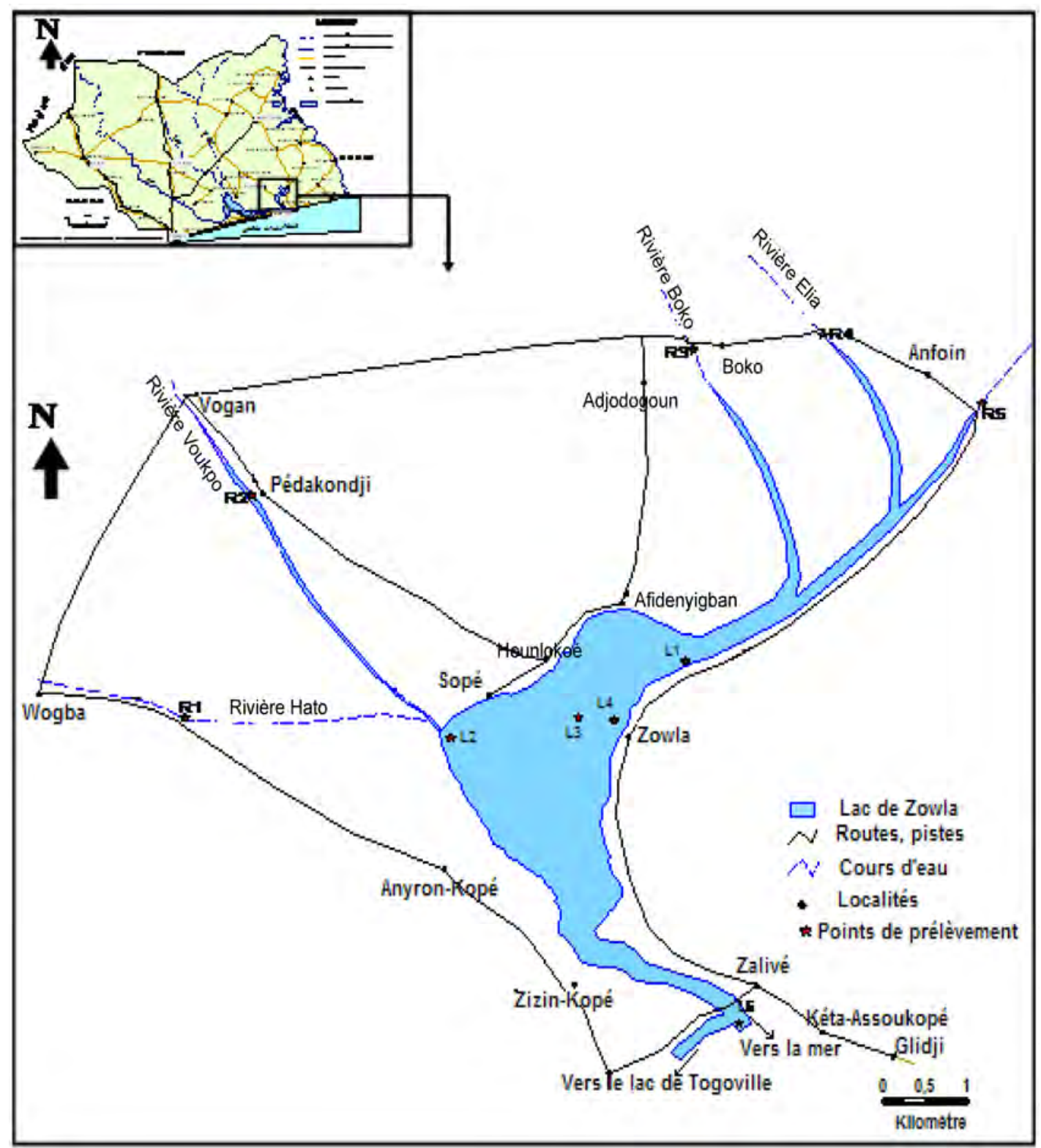

Figure 1 : Localisation de la zone d'étude.

\section{Prélèvements et méthodes d'analyse}

Les prélèvements d'eau ont été effectués en Juin 2007, correspondant à la grande saison des pluies et en Août 2007, correspondant à la petite saison sèche. La profondeur de prélèvement est de $20 \mathrm{~cm}$ environ. Pour les analyses physico-chimiques, des échantillons d'eau ont été prélevés dans des bouteilles plastiques de 1,5 litre. La température, la conductivité électrique, le $\mathrm{pH}$ de l'eau et l'oxygène dissous ont été mesurés sur site. Les autres paramètres physicochimiques ont été déterminés au laboratoire. Pour les analyses, les méthodes AFNOR (1997) ont été utilisées (Tableau 1). 
Tableau 1 : Paramètres physico-chimiques, méthodes d'analyses et équipements.

\begin{tabular}{|c|c|c|}
\hline Paramètres & Méthodes & Equipements \\
\hline $\mathrm{pH}$ & Electrométrie & pH-mètre WTW pH 330i \\
\hline Conductivité électrique & Conductimétrie & $\begin{array}{ll}\text { Conductimètre } & \text { WTW } \\
\text { Cond 330i } & \end{array}$ \\
\hline Oxygène dissous $\left(\mathrm{O}_{2}\right)$ & Oxymétrie électrochimique & Oxymètre WTW Oxi 340 \\
\hline Turbidité & Néphélométrie & $\begin{array}{l}\text { Turbidimètre DRT 100B, } \\
\text { modèle } 20012\end{array}$ \\
\hline Bicarbonate $\left(\mathrm{HCO}_{3}^{-}\right)$ & Acidimétrie & - \\
\hline Magnésium $\left(\mathrm{Mg}^{2+}\right)$ & Complexométrie EDTA & - \\
\hline Calcium $\left(\mathrm{Ca}^{2+}\right)$ & Complexométrie EDTA & - \\
\hline Oxydabilité au $\mathrm{KMnO}_{4}$ & Acide/Chaud & - \\
\hline Ammonium $\left(\mathrm{NH}_{4}^{+}\right)$ & $\begin{array}{l}\text { Spectrophotométrie } \\
\text { moléculaire }\end{array}$ & $\begin{array}{l}\text { Spectrophotomètre } \\
\text { Digitron Elvi } 675\end{array}$ \\
\hline Nitrite $\left(\mathrm{NO}_{2}^{-}\right)$ & $\begin{array}{l}\text { Spectrophotométrie } \\
\text { moléculaire }\end{array}$ & $\begin{array}{l}\text { Spectrophotomètre } \\
\text { Digitron Elvi } 675\end{array}$ \\
\hline Nitrate $\left(\mathrm{NO}_{3}{ }^{-}\right)$ & $\begin{array}{l}\text { Spectrophotométrie } \\
\text { moléculaire }\end{array}$ & $\begin{array}{l}\text { Spectrophotomètre } \\
\text { Digitron Elvi } 675\end{array}$ \\
\hline Chlorure $\left(\mathrm{Cl}^{-}\right)$ & Argentimétrie & $\begin{array}{l}\text { Spectrophotomètre } \\
\text { Digitron Elvi } 675\end{array}$ \\
\hline Sulfate $\left(\mathrm{SO}_{4}{ }^{2-}\right)$ & Néphélométrie & $\begin{array}{l}\text { Spectrophotomètre } \\
\text { Digitron Elvi } 675\end{array}$ \\
\hline Orthophosphate $\left(\mathrm{PO}_{4}{ }^{3-}\right)$ & $\begin{array}{l}\text { Spectrophotométrie } \\
\text { moléculaire }\end{array}$ & $\begin{array}{l}\text { Spectrophotomètre } \\
\text { Digitron Elvi } 675\end{array}$ \\
\hline Fer total & $\begin{array}{l}\text { Spectrophotométrie } \\
\text { moléculaire }\end{array}$ & $\begin{array}{l}\text { Spectrophotomètre } \\
\text { Digitron Elvi } 675\end{array}$ \\
\hline
\end{tabular}

La caractérisation biologique a été réalisée à travers une analyse qualitative de la flore algale. Les prélèvements ont été effectués dans des piluliers de $50 \mathrm{ml}$. Les algues ont été immédiatement fixées au lugol, gardées au frais et à l'obscurité jusqu'au laboratoire. Chaque échantillon a été laissé au repos pendant 24 heures au minimum pour permettre la sédimentation des algues. L'eau a été ensuite prélevée au-dessus du résidu algal par siphonage. La solution résiduelle concentrée en algues a été conservée dans le pilulier. Quelques gouttes de chaque échantillon ont été montées entre lame et lamelle puis observées à l'aide d'un microscope optique de marque Motic, B Series aux objectifs 10X et 40X. Pour chaque échantillon, au moins cinq lames ont été préparées afin de s'assurer de la reproductibilité des lames. Les observations ont été faites suivant des transects verticaux. Selon la morphologie générale des taxons observés, une détermination des espèces a été faite à partir des travaux de Bourrelly (1968, 1970, 1972), Komarek et Anagnostidis (1999), Lavoie et al. (2008).

\section{Analyse statistique}

Dans le but de déterminer l'influence des paramètres physico-chimiques sur la diversité du phytoplancton, une étude statistique par l'ACP (Analyse en Composantes Principales) du programme de traitement statistique Xlstat (Fahmy, 2006) a été effectuée. L'ACP est une technique mathématique qui permet de réduire un système complexe de corrélations en un 
nombre de dimensions plus faibles. Elle considère simultanément toutes les variables et leurs corrélations, révélant des corrélations qui ne sont pas souvent évidentes dans les représentations graphiques courantes ou dans les simples corrélations (Lefebvre, 1980). Les variables prises en compte dans cette étude statistique sont les paramètres physicochimiques et les classes de phytoplancton recensées dans le lac. Les pourcentages de variabilité des deux premiers facteurs F1 et F2, 52\% pour Juin et 54\% pour Août (Figures 2 et 3), ne sont pas assez importants pour permettre une bonne interprétation. Alors, les axes F1 et F3 ont été pris en compte. La matrice de corrélation (Pearson (n)) a permis de déterminer les coefficients de corrélation les plus significatifs entre les paramètres physico-chimiques et la diversité du phytoplancton.

\section{RESULTATS}

\section{Paramètres physico-chimiques}

Température, conductivité électrique et turbidité

En Juin, les températures enregistrées sont plus élevées que celles d'Août. La moyenne est de $27{ }^{\circ} \mathrm{C}$ avec un minimum de $26,9^{\circ} \mathrm{C}$ et un maximum de $27,2^{\circ} \mathrm{C}$. En Août, la moyenne est de $24{ }^{\circ} \mathrm{C}$. Le minimum est de $23,5{ }^{\circ} \mathrm{C}$ et le maximum de $24,6{ }^{\circ} \mathrm{C}$ (Tableau 2).

Pour ce qui concerne la conductivité, on remarque que ses valeurs sont plus élevées dans le lac que dans les rivières sauf au site R1 où elle atteint $7040 \mu \mathrm{s} / \mathrm{cm}$. En Juin, elle est de $1730 \mu \mathrm{s} / \mathrm{cm}$ au site L1, le plus éloigné de la mer et de $8370 \mu \mathrm{s} / \mathrm{cm}$ au site L5, plus proche de la mer (Tableau 2).

La turbidité est plus élevée en Août dans le lac. La valeur maximale enregistrée se situe au site L4 et est de 105 NTU (Tableau 2). Les valeurs les plus élevées sont enregistrées dans les rivières au site R1 en Août (163 NTU) et au site R2 en Juin (147,7 NTU).

\section{pH, alcalinité et dureté}

En Juin, l'eau du lac est presque partout neutre sauf au site L1 où elle est relativement plus acide $(\mathrm{pH}=6,6)$. Les $\mathrm{pH}$ les plus élevés sont enregistrés au site L2 et sont de 7,9 en Juin et de 7,7 en Août. Les pH enregistrés au niveau des rivières sont relativement plus faibles (Tableau 3). De la même manière, les teneurs les plus élevées en $\mathrm{HCO}_{3}{ }^{-}$dans le lac sont enregistrées au site L2 : 24,4 mg/l en Juin et $22 \mathrm{mg} / \mathrm{l}$ en Août.

Les teneurs en magnésium de Juin sont presque partout plus élevées que celles d'Août. La teneur la plus élevée en magnésium dans le lac en Juin est enregistrée au site L5 et est de $172,8 \mathrm{mg} / \mathrm{l}$. Les teneurs sont aussi plus faibles pendant la même période dans les rivières (Tableau 3).

Dans le lac, la plus forte teneur en calcium enregistrée durant les deux saisons se trouve au site L5. Elle est de $72 \mathrm{mg} / \mathrm{l}$ en Juin. La plus faible teneur $(10,4 \mathrm{mg} / \mathrm{l})$ est enregistrée en Août au même site.

Chlorures, sulfates, phosphates

$\mathrm{La}$ plus forte teneur en chlorure enregistrée dans le lac se situe en Juin au site L5 et est de $2723 \mathrm{mg} / \mathrm{l}$ (Tableau 4). La plus faible est de 132,2 mg/l au même site mais en Août. Quant aux sulfates, la plus forte et la plus faible teneur, respectivement 194,2 mg/l en Juin et $72,2 \mathrm{mg} / \mathrm{l}$ en Août (Tableau 4) ont été enregistrées au même site.

S'agissant de l'orthophosphate, dans les rivières, la valeur la plus élevée $(0,4 \mathrm{mg} / \mathrm{l})$ est enregistrée en Juin au niveau du site R2 situé sur la rivière Voukpo. Dans le lac, en Juin, le taux de phosphate est nul aux sites L1 et L5 et est plus élevé aux sites L2 et L3 (0,2 $\mathrm{mg} / \mathrm{l})$. En Août, le taux de phosphate reste élevé partout dans le lac avec la plus grande valeur $(0,4 \mathrm{mg} / \mathrm{l})$ enregistrée aux sites L2 et L5 (Tableau 4).

Eléments minéraux azotés: Ammonium $\left(\mathrm{NH}_{4}^{+}\right)$, nitrite $\left(\mathrm{NO}_{2}^{-}\right)$et nitrate $\left(\mathrm{NO}_{3}^{-}\right)$

En Juin, dans le lac, la teneur en ammonium $\left(\mathrm{NH}_{4}{ }^{+}\right)$est nulle aux sites L2 et L4 et plus élevée au site L5 avec une valeur de 
0,5 mg/l (Tableau 5). En Juin, dans le lac, la teneur en nitrite $\left(\mathrm{NO}_{2}{ }^{-}\right)$est nulle au site $\mathrm{L} 3$ et élevée au site $\mathrm{L} 2(0,9 \mathrm{mg} / \mathrm{l})$. Quant à la teneur en nitrate $\left(\mathrm{NO}_{3}{ }^{-}\right)$, dans le lac, elle est nulle aux sites L1 et L5 et plus élevée au site L2 ( 0,8 $\mathrm{mg} / \mathrm{l})$. En Août, dans le lac, la teneur en nitrite est plus faible presque partout qu'en Juin. La teneur en nitrate varie de $0,1 \mathrm{mg} / \mathrm{l}$ au site $\mathrm{L} 2$ à $1,2 \mathrm{mg} / \mathrm{l}$ au site L5 (Tableau 5).

Oxygène dissous, oxydabilité au $\mathrm{KMnO}_{4}$ et fer

En Juin, dans le lac, la teneur en oxygène dissous varie entre $3,7 \mathrm{mg} / \mathrm{l}$ au site L1 et $6,4 \mathrm{mg} / 1$ au site L2. En Août, elle se situe entre $5,1 \mathrm{mg} / 1$ au site $\mathrm{L} 5$ et 7,4 mg/l au site L2 (Tableau 6). Il y a alors eu augmentation de la teneur en $\mathrm{O}_{2}$ dissous des eaux du lac en Août par rapport à Juin sauf au site L5 (Tableau 6). Des teneurs très faibles allant jusqu'à $0,6 \mathrm{mg} / \mathrm{l}$ ont été enregistrées au site R5 en Juin et aux sites R3 et R4 en Août au niveau des rivières. En Juin, dans le lac, l'oxydabilité au $\mathrm{KMnO}_{4}$ oscille entre $5 \mathrm{mgO}_{2} / 1$ au site L5 et $9 \mathrm{mgO}_{2} / 1$ au site L3. En Août, dans le lac, elle oscille entre $6 \mathrm{mgO}_{2} / 1$ au site L2 et $10 \mathrm{mgO}_{2} / 1$ aux sites L1 et L5 (Tableau 6). La pollution organique est alors plus grande aux sites L1 et L5 en Août.
Pour ce qui est du fer, en Juin, dans le lac, sa teneur est plus faible qu'en Août sauf au site L2. Elle oscille entre $0,5 \mathrm{mg} / \mathrm{l}$ aux sites L3 et L5 et $1,1 \mathrm{mg} / \mathrm{l}$ au site L4 (Tableau 6). En Août, dans le lac, elle est relativement plus élevée au niveau de tous les sites qu'en Juin sauf au site L2. La teneur la plus élevée dans le lac est enregistrée au site L5.

\section{Etat de la biodiversité phytoplanctonique}

36 espèces sont recensées en Juin dans les eaux du lac et des rivières. Les espèces de la classe des diatomophycées sont plus diversifiées et représentent $80,55 \%$ du peuplement phytoplanctonique (Tableau 7). En Août, le nombre d'espèces est passé de 36 à 40. Certaines de ces espèces sont représentées sur la Planche 1. La proportion des diatomophycées dans le peuplement algal a diminué. Elle est passée à $40 \%$ et reste cependant la plus forte. Des espèces d'autres classes sont apparues. Il s'agit des chrysophycées et des dinophycées. Les deux saisons prises ensemble, la communauté phytoplanctonique du lac et des rivières est composée de sept classes. Parmi elles, celle des diatomophycées est la plus représentative en terme de nombre d'espèces.

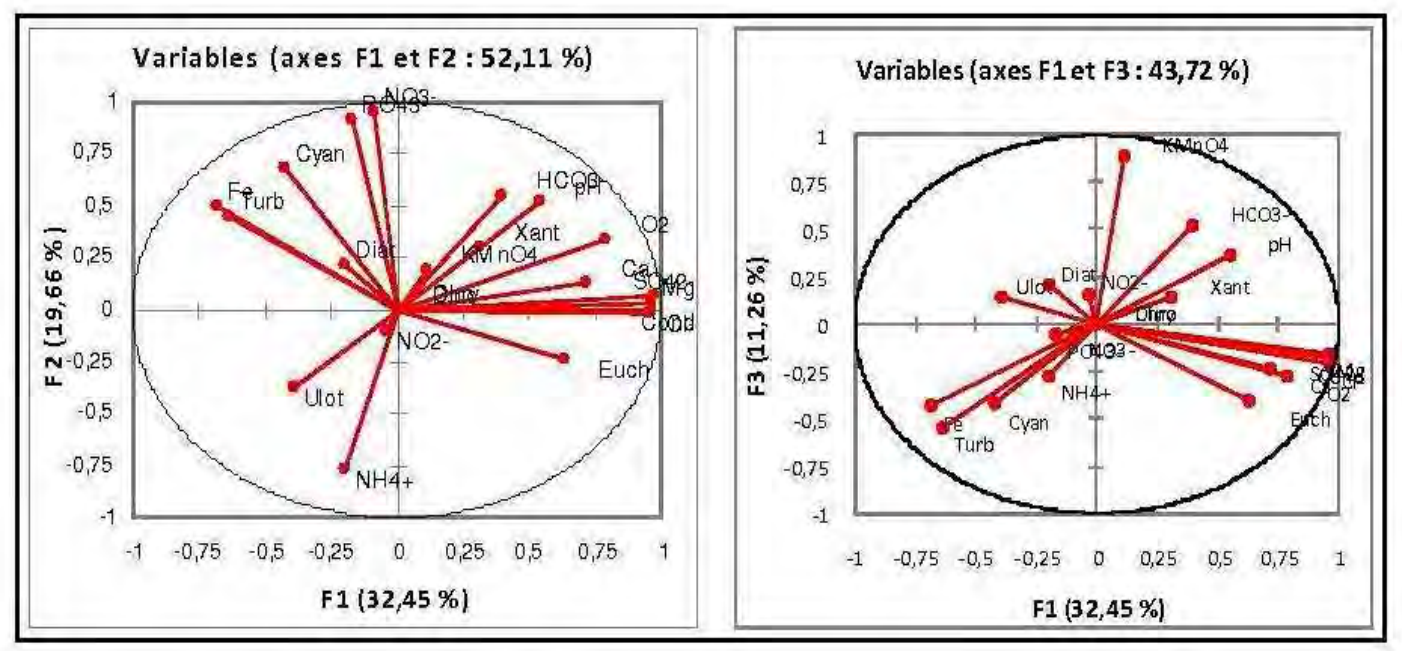

Figure 2: Diagramme des variables du mois de Juin 2007. 


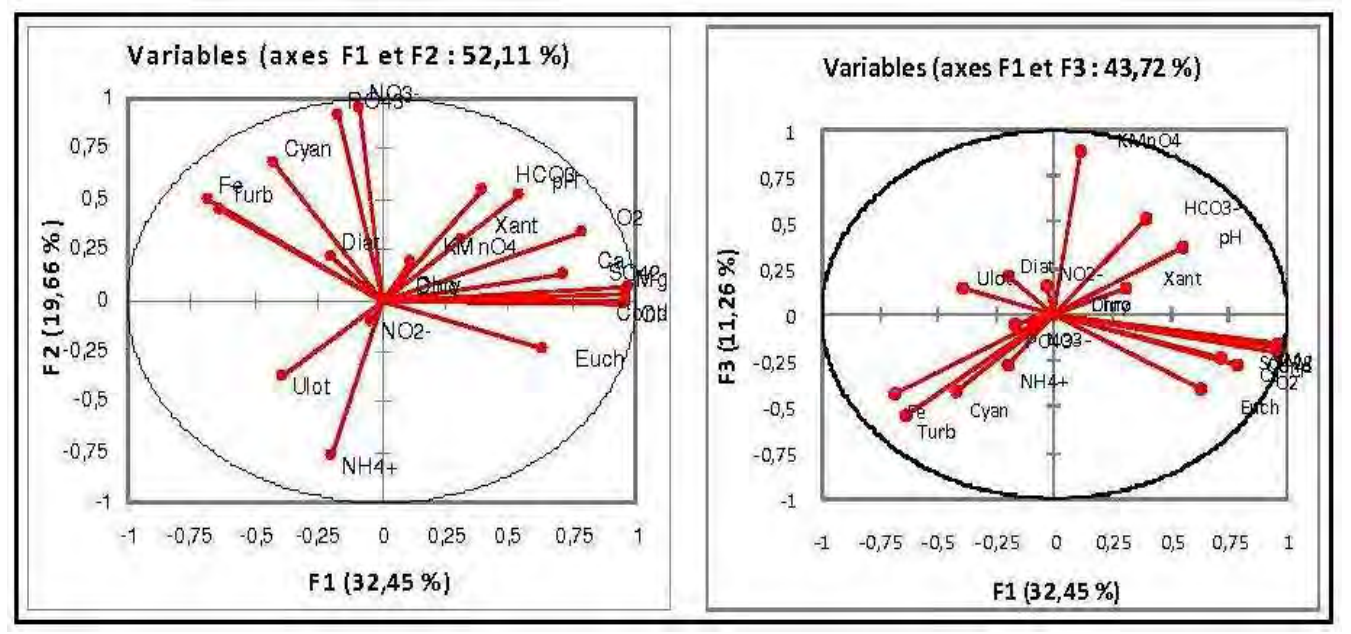

Figure 3: Diagramme des variables du mois d'Août 2007.

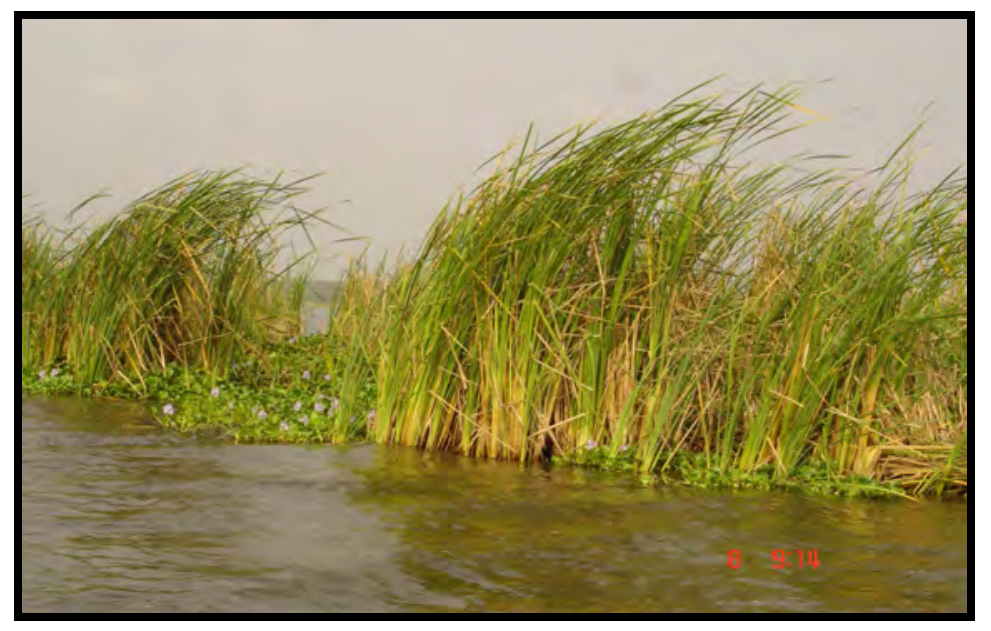

Figure 4 : Diagramme des variables du mois d'Août 2007.

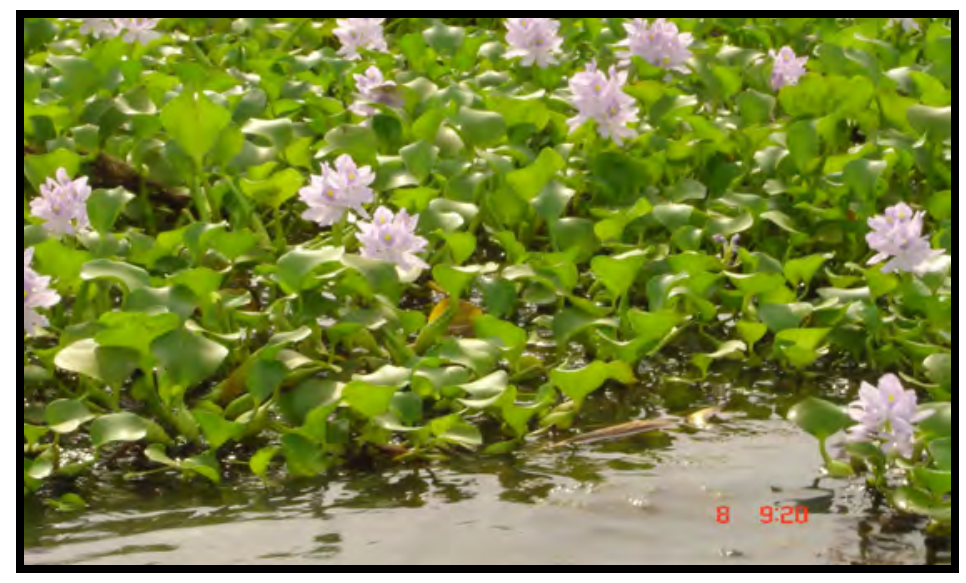

Figure 5 : Typha australis dans le lac au niveau du site L1à Zowla en Juin. 


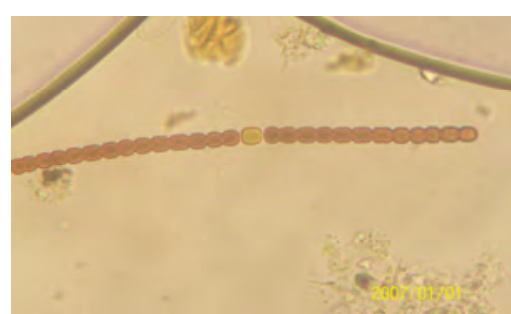

Anabaena affinis (cyanophycée)

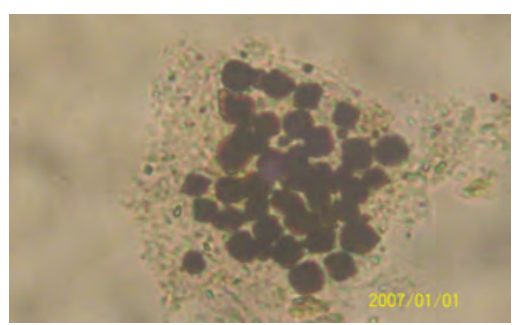

Microcystis aeroginosa (cyanophycée)

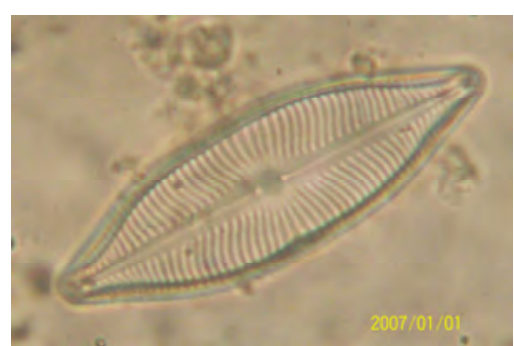

Navicula placentula (diatomophycée)

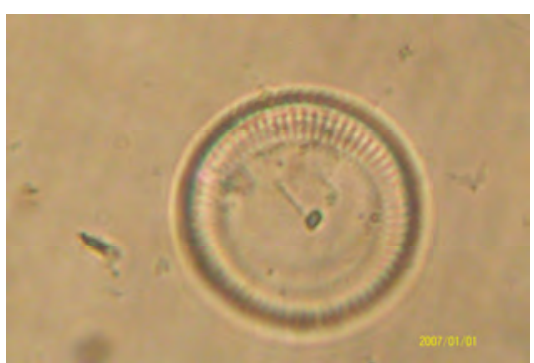

Cyclotella comta (diatomophycée)

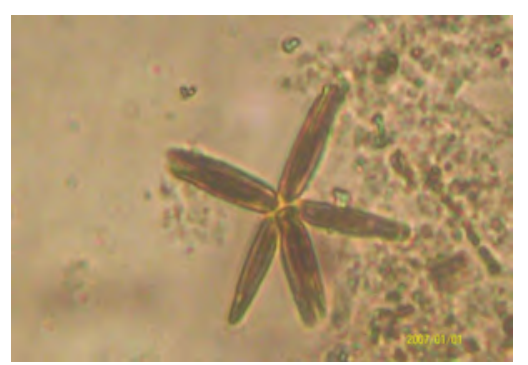

Actinastrum hantzschii (euchlorophycée)

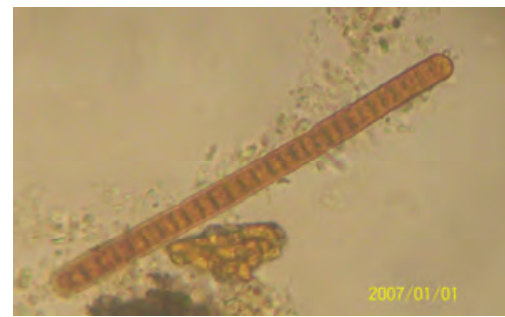

Lyngbya muralis (cyanophycée)

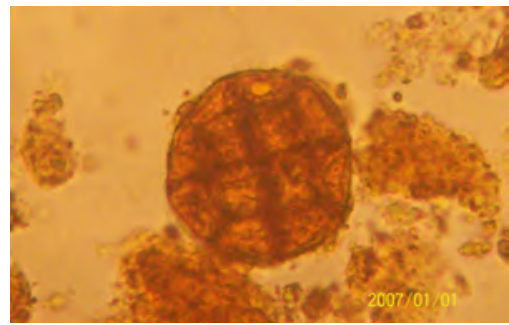

Peridinium sp (dinophycée)

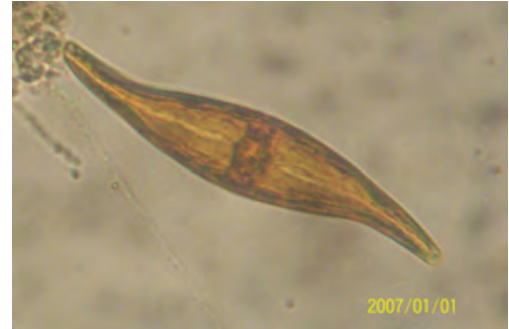

Gyrosigma sp (diatomophycée)

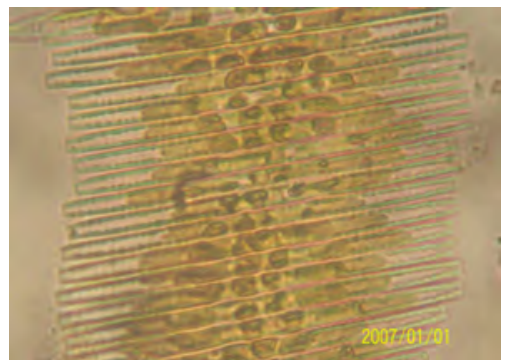

Fragilaria virescens (diatomophycée)

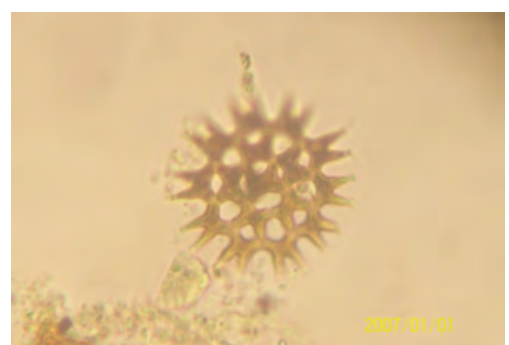

Pediastrum tetras (euchlorophycée)

Planche 1 : Quelques espèces phytoplanctoniques identifiées. 
Tableau 2 : Valeurs des températures, des conductivités et des turbidités.

\begin{tabular}{lcccccc}
\hline \multirow{2}{*}{ Echantillons } & \multicolumn{2}{c}{ Température $\left({ }^{\circ} \mathbf{C}\right)$} & \multicolumn{2}{c}{ Conductivité $(\boldsymbol{\mu}$ s/cm) } & \multicolumn{2}{c}{ Turbidité $(\mathbf{N T U})$} \\
\cline { 2 - 7 } & Juin & Août & Juin & Août & Juin & Août \\
\hline R1 & 27,0 & 23,5 & 3440 & 7040 & 50,5 & 163,0 \\
R2 & 26,9 & 24,1 & 406 & 890 & 147,7 & 7,5 \\
R3 & 26,9 & 23,5 & 418 & 476 & 12,1 & 21,5 \\
R4 & 27,2 & 23,7 & 283 & 347 & 93,0 & 10,3 \\
R5 & 27,0 & 24,1 & 477 & 449 & 82,8 & 40,0 \\
L1 & 26,8 & 24,2 & 1730 & 2960 & 12,7 & 39,5 \\
L2 & 27,0 & 24,1 & 3360 & 3560 & 45,9 & 61,2 \\
L3 & 26,8 & 24,1 & 4260 & 3610 & 27,9 & 97,9 \\
L4 & 26,9 & 24,6 & 4960 & 1291 & 45,0 & 105,0 \\
L5 & 27,0 & 24,2 & 8370 & 572 & 24,0 & 104,0 \\
\hline
\end{tabular}

Tableau 3 : Valeurs des $\mathrm{pH}$, de l'alcalinité $\left(\mathrm{HCO}_{3}{ }^{-}\right)$et des duretés (calcique et magnésien).

\begin{tabular}{lcccccccc}
\hline \multirow{2}{*}{ Echantillons } & \multicolumn{2}{c}{$\mathbf{p H}$} & \multicolumn{2}{c}{$\mathbf{H C O}_{\mathbf{3}}{ }^{-}(\mathbf{m g} / \mathbf{l})$} & \multicolumn{2}{c}{$\mathbf{C a}^{2+}(\mathbf{m g} / \mathbf{l})$} & \multicolumn{2}{c}{$\mathbf{M g}^{2+}(\mathbf{m g} / \mathbf{l})$} \\
\cline { 2 - 9 } & Juin & Août & Juin & Août & Juin & Août & Juin & Août \\
\hline R1 & 5,3 & 5,7 & 4,9 & 7,3 & 100,0 & 120,0 & 64,8 & 148,8 \\
R2 & 6,7 & 7,2 & 15,9 & 30,5 & 40,0 & 28,0 & 9,6 & 19,2 \\
R3 & 6,4 & 6,5 & 15,9 & 25,6 & 20,0 & 40,0 & 12,0 & 7,2 \\
R4 & 6,3 & 6,2 & 8,5 & 12,2 & 3,2 & 7,2 & 5,3 & 6,2 \\
R5 & 6,2 & 5,9 & 14,6 & 12,2 & 15,2 & 15,2 & 10,1 & 7,7 \\
L1 & 6,6 & 7,4 & 13,4 & 18,3 & 60,0 & 44,8 & 31,2 & 54,7 \\
L2 & 7,9 & 7,7 & 24,4 & 22,0 & 64,0 & 56,0 & 76,8 & 64,8 \\
L3 & 7,4 & 7,4 & 18,3 & 20,7 & 48,0 & 52,0 & 91,2 & 69,6 \\
L4 & 7,5 & 7,6 & 19,5 & 17,1 & 60,0 & 28,0 & 98,4 & 19,2 \\
L5 & 7,4 & 7,3 & 18,3 & 19,5 & 72,0 & 10,4 & 172,8 & 34,6 \\
\hline
\end{tabular}

Tableau 4 : Valeurs des teneurs en chlorures, en sulfates et en orthophosphates.

\begin{tabular}{lcccccc}
\hline \multirow{2}{*}{ Echantillons } & \multicolumn{2}{c}{ Chlorures (mg/l) } & \multicolumn{2}{c}{ Sulfates (mg/l) } & \multicolumn{2}{c}{ Orthophosphates (mg/l) } \\
\cline { 2 - 7 } & Juin & Août & Juin & Août & Juin & Août \\
\hline R1 & 1081,2 & 2252,5 & 173,9 & 11,2 & 0,0 & 0,3 \\
R2 & 86,1 & 214,2 & 27,3 & 0,0 & 0,4 & 0,3 \\
R3 & 95,1 & 104,1 & 0,0 & 5,3 & 0,0 & 0,2 \\
R4 & 74,1 & 86,1 & 5,3 & 0,0 & 0,0 & 0,0 \\
R5 & 134,2 & 118,1 & 1,9 & 26,4 & 0,1 & 0,1 \\
L1 & 490,5 & 861,0 & 112,9 & 129,0 & 0,0 & 0,3 \\
L2 & 1021,1 & 1041,1 & 147,6 & 133,2 & 0,2 & 0,4 \\
L3 & 1261,4 & 1081,2 & 174,8 & 129,8 & 0,2 & 0,3 \\
L4 & 1511,7 & 355,4 & 194,2 & 72,2 & 0,1 & 0,3 \\
L5 & 2723,0 & 132,2 & 272,2 & 41,7 & 0,0 & 0,4 \\
\hline
\end{tabular}


Tableau 5 : Valeurs des teneurs en ammonium, en nitrites et en nitrates.

\begin{tabular}{lcccccc}
\hline \multirow{2}{*}{ Echantillons } & \multicolumn{2}{c}{ Ammonium (mg/l) } & \multicolumn{2}{c}{ Nitrites (mg/l) } & \multicolumn{2}{c}{ Nitrates (mg/l) } \\
\cline { 2 - 7 } & Juin & Août & Juin & Août & Juin & Août \\
\hline R1 & 0,2 & 0,0 & 0,0 & 0,0 & 0,0 & 0,7 \\
R2 & 0,1 & 0,2 & 0,0 & 0,3 & 0,8 & 1,4 \\
R3 & 0,2 & 0,1 & 0,4 & 0,0 & 0,0 & 0,7 \\
R4 & 0,5 & 0,2 & 0,8 & 0,0 & 0,2 & 0,4 \\
R5 & 0,7 & 0,1 & 0,4 & 0,0 & 0,0 & 0,7 \\
L1 & 0,3 & 0,3 & 0,2 & 0,2 & 0,0 & 0,2 \\
L2 & 0,0 & 0,3 & 0,9 & 0,3 & 0,8 & 0,1 \\
L3 & 0,2 & 0,0 & 0,0 & 0,0 & 0,1 & 0,2 \\
L4 & 0,0 & 0,2 & 0,2 & 0,0 & 0,5 & 0,2 \\
L5 & 0,5 & 0,5 & 0,5 & 0,0 & 0,0 & 1,2 \\
\hline
\end{tabular}

Tableau 6 : Valeurs de l'oxydabilité au $\mathrm{KMnO}_{4}$, des teneurs en oxygène dissous et en fer total.

\begin{tabular}{lcccccc}
\hline \multirow{2}{*}{ Echantillons } & \multicolumn{2}{c}{$\left.\mathbf{K M n O}_{\mathbf{4}} \mathbf{( m g O}_{\mathbf{2}} / \mathbf{l}\right)$} & \multicolumn{2}{c}{ Oxygène dissous $(\mathbf{m g} / \mathbf{l})$} & \multicolumn{2}{c}{ Fer total $(\mathbf{m g} / \mathbf{l})$} \\
\cline { 2 - 7 } & Juin & Août & Juin & Août & Juin & Août \\
\hline R1 & 5,0 & 33,0 & 6,0 & 1,0 & 0,8 & 2,2 \\
R2 & 6,0 & 8,0 & 3,2 & 4,2 & 3,0 & 0,6 \\
R3 & 10,0 & 7,0 & 0,7 & 0,6 & 1,0 & 6,5 \\
R4 & 3,0 & 4,0 & 2,7 & 0,6 & 2,4 & 2,5 \\
R5 & 7,0 & 5,0 & 0,6 & 3,3 & 1,0 & 7,5 \\
L1 & 8,0 & 10,0 & 3,7 & 6,0 & 0,6 & 0,7 \\
L2 & 8,0 & 6,0 & 6,4 & 7,4 & 1,0 & 0,7 \\
L3 & 9,0 & 8,0 & 4,8 & 5,8 & 0,5 & 1,5 \\
L4 & 8,0 & 7,0 & 5,3 & 6,5 & 1,1 & 2,5 \\
L5 & 5,0 & 10,0 & 6,0 & 5,1 & 0,5 & 3,6 \\
\hline
\end{tabular}

Tableau 7 : Richesse spécifique des différentes classes de phytoplancton.

\begin{tabular}{lcc}
\hline \multirow{2}{*}{ Classes } & \multicolumn{2}{c}{ Pourcentage du nombre d'espèces relevées par classe } \\
\cline { 2 - 3 } & Juin & Août \\
\hline Cyanophycées & 5,56 & 10,00 \\
Xanthophycées & 5,56 & 15,00 \\
Chrysophycées & 0,00 & 5,00 \\
Diatomophycées & 80,55 & 40,00 \\
Ulothricophycées & 2,77 & 10,00 \\
Euchlorophycées & 5,56 & 10,00 \\
Dinophycées & 0,00 & 10,00 \\
\hline
\end{tabular}




\section{DISCUSSION}

\section{Paramètres physico-chimiques}

Les températures enregistrées sont partout plus basses en Août. Cela serait dû à la fraîcheur du vent de mousson qui souffle en cette période. Quant à la conductivité électrique, elle a presque partout dans le lac dépassé $1500 \mu \mathrm{s} / \mathrm{cm}$. Or, une conductivité électrique supérieure à 1500 us $/ \mathrm{cm}$ fait considérer une eau comme inutilisable dans les zones irriguées (Arrignon, 1998). L'eau du lac de Zowla n'est donc pas propice à l'arrosage des cultures maraîchères qui sont le plus souvent pratiquées le long du lac. En Août, dans le lac, elle est très faible au site L5 $(572 \mu \mathrm{s} / \mathrm{cm})$ et plus élevée au site L3 localisé au centre du lac $(3610 \mu \mathrm{s} / \mathrm{cm})$. Dans tous les cas, elle est relativement plus faible dans les rivières tributaires sauf au site R1 localisé sur la rivière Hato où elle atteint une valeur de $7040 \mu \mathrm{s} / \mathrm{cm}$ en Août. Cela serait dû à la forte minéralisation de la grande quantité de matière organique provenant des débris de macrophytes morts. En Août, au niveau des sites L5, L3 et du site L4 situé sur la berge à Zowla (Figure 1), la conductivité a chuté par rapport aux valeurs de Juin. Il y aurait alors eu une dilution occasionnée par l'entrée des eaux du fleuve Mono qui était en période de crue. Par contre, au niveau du site L1 et du site L2 situé sur la berge à l'endroit où les rivières Hato et Voukpo se jettent ensemble dans le lac, la conductivité a légèrement augmenté. Cela serait dû à une minéralisation de la matière organique provenant de la décomposition de Typha australis présent en grand nombre dans l'eau du lac à ces endroits (Figure 4).

La turbidité permettant d'évaluer la quantité de matières en suspension dans l'eau, les fortes valeurs enregistrées dans le lac en Août seraient alors dues à l'apport de sédiments dans le lac par les eaux du fleuve Mono et à la remise en suspension des particules déposées au fond par les fortes agitations de l'eau occasionnées par le vent de la mousson. En Juin, au niveau des rivières, la turbidité est élevée au site R2. Ceci serait dû au drainage des particules de phosphates par l'eau de ruissellement en pleine saison des pluies. En effet, la rivière Voukpo traverse la zone d'exploitation des phosphates du Togo et reçoit alors des eaux de ruissellement provenant de cette zone. D'ailleurs la couleur boueuse de l'eau en ce moment précis le prouve. En Août, la forte augmentation constatée au niveau du site R1 semble être due à la mise en suspension de la forte quantité de matières organiques.

En Juin, la plus faible valeur du $\mathrm{pH}$ $(6,6)$ au site L1 serait liée à la forte présence de macrophytes fixés, notamment Typha australis (Figure 4). En effet, au niveau de ce site, l'acide humique qui provient de la décomposition des macrophytes morts contribuerait à baisser le pH. Arrignon (1998) propose pour quelques groupes d'organismes aquatiques une plage de tolérance au $\mathrm{pH}$. Selon lui, la zone optimale pour la reproduction de la plupart des espèces se situe entre 6 et 7,2. En Juin et en Août, tous les sites du lac ne seraient donc pas favorables à la vie et à la reproduction des poissons sauf le site $\mathrm{L} 1$ dont le $\mathrm{pH}$ est de 6,6 (pH minimale) en Juin. Tous les sites au niveau des rivières sont favorables à la vie et à la reproduction des poissons sauf le site R1 qui a connu un $\mathrm{pH}$ de 5,3 en Juin et de 5,7 en Août. Tout comme le $\mathrm{pH}$, le $\mathrm{HCO}_{3}^{-}$présente des teneurs plus élevées au site L2 en Juin et en Août, respectivement 24,4 et $22 \mathrm{mg} / \mathrm{l}$. Ceci confirme le fait que le $\mathrm{pH}$ et les teneurs en ions $\mathrm{HCO}_{3}{ }^{-}$ seraient liés.

La teneur la plus élevée en magnésium dans le lac en Juin est enregistrée au site L5 plus proche de la mer. Etant donné que les teneurs sont aussi plus faibles pendant la même période dans les rivières, il est alors fort probable que les ions magnésium du lac soient d'origine marine. Mais, la forte teneur constatée au site R1 en Août proviendrait de la décomposition des feuilles de Typha australis très abondant autour de ce point et d'autres macrophytes, le magnésium rentrant 
dans la constitution de la chlorophylle. Quant au calcium, ses plus faibles teneurs dans le lac sont tout comme celles du magnésium enregistrées en Août au niveau de tous les sites sauf au site L3 pour le calcium et le site L1 pour le magnésium. Ceci serait dû à la dilution occasionnée par l'entrée de l'eau douce du fleuve Mono dans le lac durant ce mois.

Comme le montre le Tableau 4, on constate que les chlorures et les sulfates suivent la même évolution que la conductivité électrique et les ions magnésium dans le lac et dans les rivières que ce soit en Juin ou en Août. Les chlorures et les sulfates du lac, à l'instar du magnésium proviendraient alors de la mer.

En Juin, la teneur en phosphate étant très élevée au site $\mathrm{R} 2$, il serait alors rentré dans le lac au niveau du site L2 par la rivière Voukpo qui draine les eaux de ruissellement en provenance du site d'exploitation du minerai de phosphate. Or c'est seulement au niveau des sites L3 et L4 qui se trouvent dans le prolongement du site L2 (Figure 1) que se signale la présence de phosphate dans le lac en plus du site L2 lui-même. En Août, à part l'apport des rivières, il serait transporté dans le lac par le fleuve Mono. D'où sa teneur est élevée un peu partout dans le lac. D'après Sane (2006), dans les lacs peu profonds où l'interface eau-sédiment est bien oxygénée comme le lac de Guiers au Sénégal à l'instar du lac de Zowla, l'absorption du phosphate (orthophosphate) dans le sédiment est importante. L'essentiel du phosphate mesuré dans le lac de Zowla aurait alors une origine exogène. Le développement de l'agriculture autour du lac et des rivières tributaires devrait constituer une source importante de phosphate transporté vers le lac par les eaux de ruissellement. En Août, les fortes teneurs en phosphate occasionnées par l'apport des eaux du Mono proviendraient des engrais utilisés en agriculture et de la décomposition des déchets de toute sorte en amont et le long du fleuve Mono.
Le $\mathrm{pH}$ de l'eau du lac étant partout inférieur à 8 pendant les deux saisons (Tableau 3), l'ammonium $\left(\mathrm{NH}_{4}^{+}\right)$est alors sans danger pour la vie des organismes vivant dans l'eau. En effet, la présence des deux formes de l'azote ammoniacal $\left(\mathrm{NH}_{3}\right.$ et $\left.\mathrm{NH}_{4}{ }^{+}\right)$ est fonction du $\mathrm{pH}$. Ainsi, l'azote ammoniacal aura peu d'influence sur la faune et la flore aquatiques tant que le $\mathrm{pH}$ restera inférieur à 8 , car il est sous sa forme non toxique $\left(\mathrm{NH}_{4}{ }^{+}\right)$. Par contre, pour des $\mathrm{pH}$ plus élevés, son action sur les organismes aquatiques sera négative, car il est sous sa forme toxique $\left(\mathrm{NH}_{3}\right)$ (Bremond et Vuichard, 1973). Ceci est confirmé par Lavoie et al. (2008), selon qui le $\mathrm{NH}_{4}{ }^{+}$est toxique lorsque le $\mathrm{pH}$ est élevé, ce qui fait des nitrates une source d'azote très importante pour la croissance des algues (Wetzel et Likens, 2000).

Dans les systèmes aquatiques, la température de l'eau et la salinité influent sur le taux de dissolution de l'oxygène. D'après Tardat-Henry et Beaudry (1984), quand l'eau contient des quantités très importantes de sels, la solubilité des gaz diminue. Dans le lac de Zowla, le taux d'oxygène dissous a augmenté en Août par rapport à Juin. C'est alors la dilution des sels occasionnée par l'entrée des eaux du fleuve Mono en plus de la diminution de la température de l'eau qui auraient agi en Août. L'oxygène de l'eau provient de son contact avec l'air, sa dissolution étant facilitée par le brassage. Elle a également une origine biologique par la fonction chlorophyllienne exercée par les végétaux du périphyton, les algues planctoniques, ainsi que les phanérogames aquatiques dans les zones littorales des plans d'eau (Arrignon, 1998). Parmi les causes de variation de sa teneur, on peut citer la présence des matières organiques oxydables, des organismes, des germes aérobies, des graisses, des hydrocarbures, des détergents, ainsi que la perturbation des échanges atmosphériques à l'interface air-eau (Rodier et al., 1996). Dans les eaux douces en particulier, c'est surtout la température de l'eau qui influe sur la solubilité de l'oxygène. 
D'après la table de WINKLER (Protec Traitement des Eaux, 2002), la solubilité de l'oxygène baisse avec l'augmentation de la température. Cela pourrait constituer la raison pour laquelle dans la plupart des sites du lac, l'oxygène dissous est plus faible en Juin où les températures sont plus élevées (moyenne de température : $27^{\circ} \mathrm{C}$ ) qu'en Août où les températures de l'eau sont plus faibles (moyenne de température : $24^{\circ} \mathrm{C}$ ). S'agissant de l'oxydabilité au $\mathrm{KMnO}_{4}$, en Août, on remarque une augmentation des teneurs en matières organiques aux sites L1 et L5 par rapport aux teneurs de Juin. Cette augmentation serait due à la décomposition de Typha australis au site L1 (Figure 4) et à l'apport de la matière organique par le fleuve Mono au site L5. Selon Kenneth (1974), à $20^{\circ} \mathrm{C}$, la saturation en oxygène dans l'eau d'un cours d'eau est de $8,57 \mathrm{mg} / 1$; mais pour une eau riche en matières organiques, cette valeur peut diminuer considérablement, voire s'annuler. Selon Billen et al. (1999), la biodégradation de cette importante charge en matières organiques s'accompagne d'une consommation de l'oxygène dissous par les principaux agents dégradateurs que constituent les bactéries hétérotrophes. Dans le lac de Zowla, la teneur en oxygène dissous reste faible et oscille entre 3,7 et $6,4 \mathrm{mg} / \mathrm{l}$ pendant la grande saison pluvieuse. Donc, le lac de Zowla contiendrait de la matière organique en quantité non négligeable dont la biodégradation par les microorganismes aérobies consommerait une quantité importante d'oxygène. La forte présence d'Eichornia crassipes sur l'eau (Figure 5) peut aussi être l'une des causes de la plus faible teneur en oxygène dissous enregistrée au site L1 en Juin. Les très faibles teneurs en oxygène dissous enregistrées au niveau de presque tous les sites des rivières pendant les deux saisons seraient dues au confinement de ces milieux. Ce confinement peut occasionner un manque d'aération de ces milieux entraînant une décomposition accélérée des macrophytes présents en grand nombre. A cela s'ajouterait la consommation du peu d'oxygène disponible pour la biodégradation de l'importante charge organique provenant de la décomposition des macrophytes. Certains de ces sites ont des teneurs en oxygène dissous inférieures à $1 \mathrm{mg} / \mathrm{l}$. C'est le cas des sites R3 (0,7 mg/l en Juin ; 0,6 mg/l en Août), R4 (0,6 mg/l en Août) et R5 (0,6 mg/l en Juin). Toute forme de vie aquatique serait alors impossible au niveau de ces sites car ces valeurs sont inférieures au seuil létal de vie aquatique en oxygène dissous qui est de $1 \mathrm{mg} / \mathrm{l}$ (Hubiche, 2002).

S'agissant du fer, on constate que dans le lac, ses teneurs sont relativement plus élevées au niveau de presque tous les sites en Août qu'en Juin. En effet, le fer proviendrait du lessivage des sols ferralitiques. Il serait alors surtout apporté dans le lac en Août par l'eau du fleuve Mono et dans ce cas, il proviendrait du lessivage des terrains ferralitiques en amont et autour du fleuve.

L'influence exercée par la mer et les eaux continentales sur le lac de Zowla semble être la même pour la lagune Ebrié à Abidjan en Côte-d'Ivoire. En effet, selon Durand et Chantraine (1982), l'hydroclimat de la partie estuarienne de la lagune Ebrié et donc de la baie de Biétri évolue sous un double contrôle océanique et continental. Lorsque les apports continentaux sont maximaux, l'influence marine est fortement réduite. Inversement, lors de l'étiage du fleuve, l'influence océanique prédomine. Au Sénégal, d'après les travaux de Sane (2006), le lac de Guiers, avant 1987 était soumis à l'influence de la mer et du fleuve Sénégal. Ici, contrairement au lac de Zowla, l'intrusion de la mer se fait par remontée des eaux salées vers le lac via le fleuve suite à l'étiage de ce dernier. Mais avec la construction et la mise en service du barrage antisel de Diama en 1985 et du barrage hydro-électrique de Manantali au Mali en 1987, dont le rôle est de réguler l'intensité de la crue du fleuve Sénégal, le fonctionnement du lac est devenu totalement 
contrôlé. Les intrusions salines sont arrêtées, et les eaux sont douces en permanence.

\section{Corrélation entre diversité phytoplanctonique et paramètres physico- chimiques}

Pour ce qui concerne la corrélation entre les paramètres physico-chimiques et la diversité phytoplanctonique, le diagramme des variables de par les axes F1 et F2 donne en Juin les corrélations suivantes confirmées par les coefficients de corrélation. Les cyanophycées sont corrélées avec le fer ( $\mathrm{r}=$ $0,766)$, les orthophosphates $(r=0,780)$, les nitrates $(r=0,629)$ et la turbidité $(r=0,772)$. Les cyanophycées sont alors mieux diversifiées dans les milieux turbides riches en fer, en orthophosphate et en nitrate. La forte corrélation entre le fer et la turbidité ( $\mathrm{r}=$ $0,897)$ montre que la turbidité de l'eau serait due à la forte teneur en fer à l'état particulaire. La grande richesse spécifique des cyanophycées dans les milieux turbides du lac et des rivières tributaires riches en orthophosphates et en nitrates est en adéquation avec les travaux de Sanaa (2006) selon qui les cyanophycées expriment leur dominance prodigieuse en effectifs et en espèces dans les eaux les plus riches en nutriments, particulièrement l'azote ammoniacal $\left(\mathrm{NH}_{4}^{+}\right)$, en matières en suspension (MES) et organiques particulaires (MOP). Les euchlorophycées sont corrélées avec la conductivité $(\mathrm{r}=0,745)$, le magnésium $(r=0,748)$, les chlorures $(r=0,770)$ et les sulfates $(r=0,584)$. Elles sont alors mieux diversifiées dans les milieux très minéralisés. Les diatomophycées ne sont corrélées avec aucun paramètre physico-chimique ( $\mathrm{r}$ faibles). Cependant, elles sont les plus représentatives en terme de nombre d'espèces. Ceci montre encore une fois que ce sont des espèces cosmopolites (Lavoie et al., 2008).

En Août, les axes F1 et F3 montrent une corrélation entre les xanthophycées et l'ammonium $(r=0,680)$. Ces mêmes axes ont permis de déterminer des corrélations entre les chrysophycées et les bicarbonates $(r=0,579)$; les chrysophycées et les nitrites $(r=0,606)$; les chrysophycées et les nitrates $(r=0,641)$. Les xanthophycées sont alors plus spécifiquement riches dans les milieux aquatiques riches en ammonium, les chrysophycées le sont pour les bicarbonates, les nitrites et les nitrates. Il y a aussi une corrélation forte entre les cyanophycées et l'ammonium ( $\mathrm{r}=0,748)$, ce qui est aussi conforme à la première conclusion de Sanaa (2006) énoncée plus haut. Les deux diagrammes des variables affichent une corrélation entre les ulothricophycées et les bicarbonates $(r=0,641)$.

Dans la présente étude sur le lac de Zowla et ses tributaires, ce sont les diatomophycées qui prédominent. Mais dans plusieurs lacs en Afrique de l'Ouest, ce sont les cyanophycées et les euchlorophycées qui dominent. C'est le cas des études sur le lac de Guiers au Sénégal par Sane (2006), Carl Bro International (2000). Cette différence entre ce lac et celui de Zowla est due à l'arrêt des eaux marines par la construction en 1985 du barrage anti-sel de Diama sur le fleuve Sénégal (Sane, 2006). Ce qui n'est pas le cas du lac de Zowla.

\section{Conclusion}

Le lac de Zowla est donc soumis à un double contrôle océanique et continental. Les paramètres physico-chimiques de l'eau du lac de Zowla, et par conséquent le phytoplancton, sont influencés en grande saison des pluies (Juin) par la mer et en petite saison sèche (Août) par le fleuve Mono. Le type de pollution révélé par les études est la pollution minérale plus accentuée en grande saison pluvieuse. Elle est causée par les sels dissous en provenance de la mer (magnésium, sulfate, chlorure). Les faibles teneurs en orthophosphates proviendraient de la zone d'exploitation du minerai de phosphate au Togo et des engrais phosphatés utilisés en agriculture. Quant à la pollution organique, 
elle est beaucoup plus grande dans le lac en Août.

S'agissant du phytoplancton, au total 68 espèces regroupées dans six classes ont été recensées. Mais la classe des diatomophycées reste la plus représentative en terme de nombre d'espèces durant les deux saisons. L'analyse en Composantes Principales (ACP) a montré que les cyanophycées sont plus diversifiées en espèces dans les milieux turbides riches en fer, en orthophosphate, en nitrate et en ammonium. Les euchlorophycées le sont dans les milieux très minéralisés, c'està-dire riches en magnésium, chlorure, sulfate et de conductivité électrique très grande et les xanthophycées dans les milieux aquatiques riches en ammonium. Les chrysophycées le sont dans les milieux riches en bicarbonate, nitrites et nitrates et les ulothricophycées dans les milieux riches en bicarbonates. Quant aux diatomophycées, elles ne sont corrélées avec aucun paramètre physico-chimique. Mais, étant donné qu'elles sont les plus représentatives en terme de nombre d'espèces, elles sont alors des espèces cosmopolites.

\section{REFERENCES}

AFNOR. 1997. Qualité de l'Eau (Tome 2), Méthodes d'Analyse 1 : Analyse Organoleptique, Mesures PhysicoChimiques, Paramètres Globaux, Composés Organiques ( 2 édn). AFNOR: Paris.

Arrignon J. 1998. Aménagement Piscicole des Eaux Douces ( $5^{\mathrm{e}}$ édition). Lavoisier Tec. Doc: Paris.

Billen G, Garnier J, Servais P, Brion N, Ficht A, Even S, Berthe T, Poulin M. 1999. L'Oxygène: un Témoin $d u$ Fonctionnement Microbiologique. Ifremer : Paris

Bourrelly P. 1968. Les Algues Jaunes et Brunes (Tome II). Ed. Boubée: Paris; $438 \mathrm{p}$.

Bourrelly P. 1970. Les Algues Bleues et Rouges (Tome I). Ed. Boubée: Paris; $511 \mathrm{p}$.
Bourrelly P. 1972. Les Algues Vertes (Tome III). Ed. Boubée: Paris ; 572 p.

Bremond R, Vuichard R. 1973. Paramètres de la qualité des eaux. Documentation Française, 178 p.

Carl Bro International. 2000. Étude bathymétrique et limnologique du lac de Guiers. Rapport de synthèse, Hydroconsult. International, SGPRE, $119 \mathrm{p}$.

Chikhaoui MA, Hlaili AS, Mabrouk HH. 2008. Réponses saisonnières du phytoplancton aux rapports d'enrichissements N:Si:P dans la lagune de Bizerte (Sud-Ouest de la Méditerranée). Comptes Rendus Biologies, 331: 389-408.

Durand JR, Chantraine JM. 1982. L'environnement climatique des algues ivoiriennes. Rev. Hydrobiol. Trop., 15: 85-113.

Edorh T, Blivi A, Bandjé A, Abotsi K. 2008. Présence d'algues toxiques dans les eaux marines et saumâtres du littoral togolais. Annales des Sciences Agronomiques du Bénin, 10(2): 165-177.

Fahmy T. 2006. www.xlstat.com.Addinsoft, 1993-2006.

Gulati RD, Van Donk E. 2002. Lakes in the Netherlands, their origin, eutrophication and restoration: state- of- the- art review. Hydrobiologia, 478: 73-106.

Hubiche J-L. 2002. Cahier 2002 Indicateurs. Loire Estuaire Cellule de Mesures et de Bilans, Nantes.

Jiang J-G, Shen Y-F. 2006. Estimation of the natural purification rate of a eutrophic lake after pollutant removal. Ecological Engineering, 28: 166-173.

Kenneth M. 1974. La Biologie de la Pollution. Imprimerie Bayeusaine : France ; 26-35; 63-67.

Komarek J, Anagnostidis K. 1999. Süsswasserflora von Mitteleuropa 19/1. Cyanoprokaryota1.Teil: Chroococcales. Gustav Fischer : Stuttgart ; 548 p. 
Lavoie I, Hamilton PB, Campeau S, Grenier M, Dillon PJ. 2008. Guide d'Identification des Diatomées des Rivières de l'Est du Canada. Presse de l'Université du Québec ; 241 p.

Lefebvre JC. 1980. Introduction to Multidimensionnal Statistical Analysis ( $2^{\text {nd }}$ edn). Masson : Paris ; 259p.

Protec Traitement des Eaux. 2002. Solubilité de l'oxygène en fonction de la température. http://perso.wanadoo.fr/ protec/22solubilitédel'oxygène.htm, p. 22.

Rodier J, Bazin C, Broutin J-P, Chambon P, Champsaur H, Rodi L. 1996. L'Analyse de l'Eau : Eau Naturelle, Eau Résiduaire, Eau de Mer ( $8^{\mathrm{e}}$ édn). DUNOD : Paris ; $1384 \mathrm{p}$.

Sanaa B. 2006. Structure, dynamique et typologies physico-chimiques et phytoplanctoniques de l'estuaire du Bou
Regreg (Côte atlantique marocaine). Thèse de Doctorat, Université Mohammed V-Agdal de Rabat (Maroc), $252 \mathrm{p}$.

Sane S. 2006. Contrôle environnemental de la production primaire du lac de Guiers au Nord du Sénégal. Thèse de Doctorat, Université Cheikh Anta Diop de Dakar, Sénégal, 187 p.

Tardat-Henry M, Beaudry JP. 1989. Chimie des Eaux. Les éditions le Griffon d'Argile Inc. : Canada ; 314 p.

Wetzel RG, Likens GE. 2000. Limnological Analyses ( $3^{\text {rd }}$ edn). Springer-Verlag Inc.: New-York; 429 p.

Xie L, Xie P. 2002. Long-term dynamics of phosphorus in a shallow, subtropical chinese lake with the possible effects of cyanobacterial blooms. Water Res., 36: 343-349, 1956-1999. 